\title{
BMJ Open Exploring the impact of the COVID-19 pandemic on doctors' core workplace needs: a qualitative study of internal medicine trainees in Scotland
}

\author{
Joanne Kerins (D) , ${ }^{1,2}$ Ailsa Lauren Hamilton, ${ }^{3}$ Jemma Pringle, ${ }^{4}$ Fiona Farquhar, ${ }^{5}$ \\ Victoria Ruth Tallentire ${ }^{1,3,4}$
}

To cite: Kerins J, Hamilton AL, Pringle J, et al. Exploring the impact of the COVID-19 pandemic on doctors' core workplace needs: a qualitative study of internal medicine trainees in Scotland. BMJ Open 2021;11:e053506. doi:10.1136/ bmjopen-2021-053506

- Prepublication history for this paper is available online. To view these files, please visit the journal online (http://dx.doi. org/10.1136/bmjopen-2021053506).

Received 14 May 2021 Accepted 16 June 2021

D) Check for updates

(C) Author(s) (or their employer(s)) 2021. Re-use permitted under CC BY-NC. No commercial re-use. See rights and permissions. Published by BMJ.

${ }^{1}$ Scottish Centre for Simulation and Clinical Human Factors, Larbert, UK

${ }^{2}$ Acute medicine, NHS Greater Glasgow and Clyde, Glasgow, UK ${ }^{3}$ The University of Edinburgh,

Edinburgh, UK

${ }^{4}$ NHS Education for Scotland,

Edinburgh, UK

${ }^{5}$ Acute medicine, NHS

Lanarkshire, Bothwell, UK

Correspondence to

Dr Joanne Kerins;

joanne.kerins@ggc.scot.nhs.uk

\section{ABSTRACT}

Objectives This study aimed to explore how the COVID-19 pandemic has impacted the workplace core needs of internal medicine (IM) trainees in Scotland.

Design This qualitative study used an observational approach of interprofessional workshops combined with subsequent individual interviews with IM trainees. Workshops and interviews were audiorecorded, transcribed verbatim and analysed utilising NVivo software. Template analysis was used with the Autonomy/control, Belonging and Competence $(A B C)$ of doctors' core needs outlined in the 2019 General Medical Council report Caring for doctors, caring for patients as a conceptual lens for the study.

Setting The national IM boot camp in Scotland includes a 2-hour interprofessional workshop which is trainee led and explores current challenges in the workplace, including the impact of the pandemic on such relationships.

Participants Twelve workshops, involving 72 trainees, were included with ten trainees taking part in the subsequent interview process. Trainees representing all four regions in Scotland were involved.

Results Trainees described all core needs having been impacted by the pandemic. They described a loss of autonomy with emergency rotas but also through a pervasive sense of uncertainty. The data revealed that work conditions improved initially with additional resources which have since been removed in some areas, affecting trainees' sense of value. Analysis found that belonging was affected positively in terms of increased camaraderie but also challenged through inability to socialise. There were concerns regarding developing competence due to a lack of teaching opportunities.

Conclusions Using the $\mathrm{ABC}$ of doctor's core needs as a conceptual framework for this study highlighted the impact of the COVID-19 pandemic on all domains for IM trainees in Scotland. It has highlighted an opportunity to foster the renewed sense of camaraderie among healthcare teams, while rebuilding work conditions to support autonomy and competence.

\section{INTRODUCTION}

It is well recognised that the COVID-19 pandemic has impacted the training of junior doctors, with over $80 \%$ of doctors in
Strengths and limitations of this study

- This qualitative study explores the perspectives of a national cohort of internal medicine trainees during the COVID-19 pandemic, the impact on whom has been less represented to date.

- The data analysis incorporated a framework applicable to all doctors, the Autonomy/control, Belonging and Competence $(\mathrm{ABC})$ of core workplace needs, increasing transferability of results to doctors training in other specialties.

- In-depth interviews and observational data from an interprofessional workshop offered a unique insight into the personal experiences and reflections of medical trainees.

- The study is limited to the reflections of internal medicine trainees, and therefore, does not directly capture the disruption that is likely to have been encountered across all specialties.

training reporting disruption caused by the pandemic in their General Medical Council (GMC) national training survey in $2020 .{ }^{1}$ The pandemic has resulted in increased demand on the National Health Service (NHS), with the UK being one of the most affected countries in Europe. ${ }^{2}$ The combination of disrupted training and increased patient demand have caused concern relating to the well-being of trainees. ${ }^{3} 4$ The introduction of social distancing guidance and personal protective equipment has altered the workplace environment for all healthcare workers in the UK. There have been some 'silver linings' described with new virtual training methods and a transition to online outpatient clinics. ${ }^{5}$ There is evidence of innovation and adaptability, finding opportunity in crisis during the pandemic, which indicates an ability to make positive change which should be harnassed for the future. ${ }^{6-10}$

Comparisons have been drawn between the impact of the COVID-19 pandemic and 
the various phases of disaster with associated emotions. ${ }^{11}$ This includes a heroic phase at the outset with a honeymoon phase of community cohesion and related peaks of emotional highs. ${ }^{12}{ }^{13}$ This is followed by a period of disillusionment where emotions are are low and gradually improve with progress into a reconstruction phase. ${ }^{14} 15$ The phase of reconstruction involves taking responsibility for rebuilding with a new beginning and it is crucial that we understand the challenges and opportunities navigated throughout the pandemic in order to do this successfully. In rebuilding, this includes appreciating the ways in which the workplace changes and training impacts have affected trainees, both positively and negatively, so as to move forward effectively.

In considering the needs of trainee doctors in the UK, the GMC have described the Autonomy/control, Belonging and Competence (ABC) of doctors' core needs in their 2019 report Caring for doctors, caring for patients. ${ }^{16}$ This report set out to review the factors that impact on the mental health and well-being of doctors, with the aim of improving the culture and working environments for doctors. The core needs identified include $\mathrm{ABC}$ with subcategories therein and descriptions as outlined in table $1 .{ }^{16}$ This key report discussed immediate steps and calls to action with the intention of improving UK healthcare environments to support doctors in caring for patients. ${ }^{16}$ Since its publication, however, the COVID-19 pandemic has transformed the clinical workplace unexpectedly and we must consider how these changes have impacted the core needs identified in the GMC report. Throughout the heroic and honeymoon phase, it is possible that some of the suggested changes from the GMC report were accelerated, while we know that some of them will have been postponed, and others likely neglected. Revisting these core needs after the disruption caused by the pandemic is an opportune time to address what we can learn from the pandemic going forward.

This study aimed to explore the ways in which COVID-19 pandemic has impacted the core workplace needs of internal medicine (IM) trainees in Scotland.

\section{METHODS}

\section{Context}

IM Training is a 3-year training programme for junior doctors in the UK wishing to pursue a career in medical specialties. In Scotland, simulation training is integrated into each year of the training programme which includes a 3-day IM boot camp within the first year. Between August and December 2020, the IM boot camp was delivered to 90 IM trainees at the Scottish Centre for Simulation and Clinical Human Factors. IM trainees participated in a 3-day boot camp which included an interprofessional communication workshop that explored challenges and coping strategies. Trainees took part in the 2-hour workshop in groups of six with two facilitators (JP and FF). The discussion was trainee-led covering areas of particular challenge over recent months, including the effects of the COVID-19 pandemic on their workplace experience.

\section{Conceptual framework}

The conceptual framework for this study is the $\mathrm{ABC}$ of doctor's core needs defined as $\mathrm{ABC}$, as described in table 1 .

\section{Data collection}

The first stage of this study utilised an observational approach, audiorecording the workshops in which all participants had consented. This approach was chosen to gain insight into participants' accounts of experiences while not influencing their learning event. ${ }^{17}$ Subsequently, consenting participants were contacted by email 3-6 months following their boot camp and invited to an individual interview via Microsoft Teams, conducted by $\mathrm{JK}$, to further explore experiences. JK is an acute medicine doctor with 8 years of postgraduate clinical training and experience of medical education research. Audiorecordings of both workshops and interviews were anonymised and transcribed verbatim.

\section{Data analysis}

Transcripts were independently analysed by JK and ALH using template analysis and utilising NVivo software. ALH is a clinical fellow in medical education with 8 years of postgraduate clinical training currently undertaking an doctoral research degree in medical education. In template analysis, a template based on prior research is applied and the initial template may be modified by the data with new codes added inductively. ${ }^{18}$ This constructivist study used the GMC report's ABC of doctors' core needs as an initial coding framework. ${ }^{16} \mathrm{JK}$ and ALH met on a regular basis and discussed each category of the framework in detail and compared coding. Disagreements on coding were discussed with reference to the $\mathrm{ABC}$ of doctors' core needs framework, ${ }^{16}$ with final decisions on analysis made by JK. The resultant framework is therefore her conceptualisation of the framework produced by the interactions between $\mathrm{JK}$, the research participants and her coresearchers.

\section{Patient and public involvement}

Patients and/or the public were not involved in the design, conduct, reporting or dissemination of this study.

\section{RESULTS}

Twelve workshops, involving 72 trainees, were included in the analysis. This represents the number of IM trainees present in workshops where all partipating trainees had consented. Workshops lasted 2 hours with trainees aged between 24 and 35 years, with 34 trainees identifying as female and 38 identifying as male. Trainees from all four regions of Scotland (West, South East, East and North) were included. Ten trainees took part in the subsequent interview process, including seven identifying as female and three identifying as male. Trainees were aged 24-35 


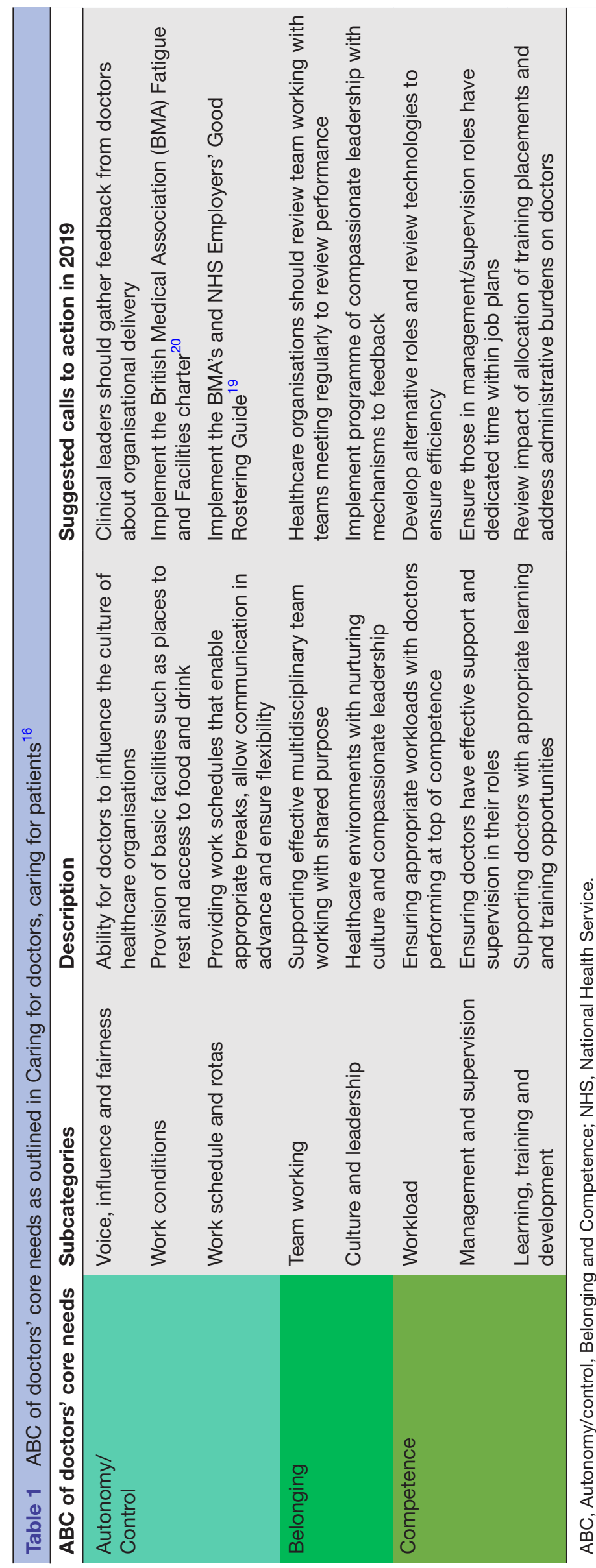




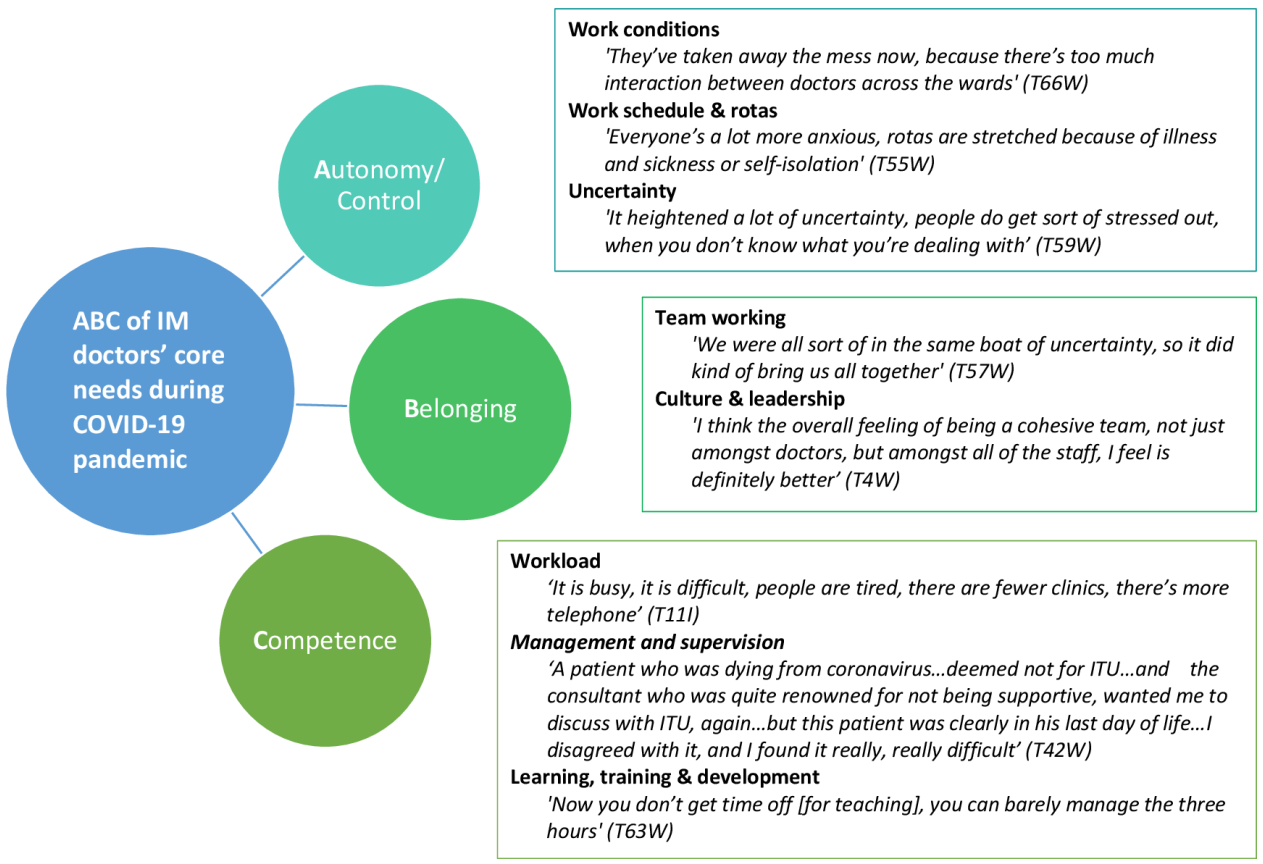

Figure $1 \mathrm{ABC}$ of IM trainee doctors' core needs during COVID-19 pandemic with illustrative quotes. ABC, Autonomy/control, Belonging and Competence; IM, internal medicine.

years, again from all four regions in Scotland. Interviews lasted between $18 \mathrm{~min}$ and $35 \mathrm{~min}$ (average of $28 \mathrm{~min}$ ).

Trainees described all core needs having been impacted by the pandemic, as summarised in figure 1. Quotes taken from the workshops are indicated by a 'W' after the trainee code, and those taken from the interviews are indicted by an 'I'. The ABC framework and subheadings are described below. ${ }^{16}$

\section{Autonomy/Control}

Work conditions

Trainees reflected on some of the benefits that the COVID-19 pandemic brought to the work conditions:

They employed a psychologist, they had a relaxation area, there was hot drinks on tap...I felt like it made life so much easier, because the care that was in place for staff just increased massively, and it was a really stressful time and no one knew what was happening but there were places you could go that were really nice, and it just felt so nice that the NHS was doing that. (T5W)

They found that 'having a non-clinical area to take breaks in' (T3W) was very beneficial because 'if you have to go somewhere on the ward or unit to sit and have your lunch, people can just find you and you don't actually get that detachment' (T4W). However, some trainees felt undervalued as such designated areas had already been disbanded or were planned to be. They found that 'it's when life goes back to more normal, it got lost a little bit' (T57W). Trainees recognised the importance of having appropriate rest areas as they reflected 'we need to be healthy too, working' (T6W).

\section{Work schedules and rotas}

Given the evolving pandemic, trainees found that 'shifts were changing and there was a lot of rota gaps' (T40I). The prolonged nature of the pandemic was taking its toll: 'certainly at the start because there were these extra shifts that needed covered...I think in the first peak of COVID-19 a lot of people volunteered for these shifts more regularly. I think a lot of people are getting fatigued and burnt out and the take up of it is less' (T61W). Their experience was not in keeping with the British Medical Association (BMA) and NHS Employer's Good Rostering Guide which was a key action point from the GMC report in 2019. ${ }^{16} 19$

\section{Uncertainty}

In contrast with the 'Voice, influence and fairness' subheading of the $\mathrm{ABC}$ framework ${ }^{16}$ within autonomy and control, whereby doctors can influence the culture of their healthcare organisations, the trainees described a resounding sense of uncertainty in relation to the COVID-19 pandemic. There was a loss of control due to the pervasive uncertainty, not only due to work conditions and rotas but due to the pandemic in general as 'it was a really stressful time, and no one knew what was happening' (T5W). This was particularly relevant in the initial stages:

When COVID started, it wasn't clear what was going on... and we were obviously panicking. (T44W)

There was uncertainty around the management of patients and changing protocols:

Initial phases when there was a lot of uncertainty with protocols, it actually caused quite a lot of stress. (T59W) 
There was also concern around transmission inside and outside the workplace:

Worried about spreading it...or worried about catching it...or worried about spreading it to their family or bringing it into the hospital. (T55W)

Overall trainees reflected that the burden of uncertainty affected them in a negative manner.

\section{Belonging}

Team working

Trainees found there were challenges with team working in line with social distancing requirements as:

You would always mix in the mess [doctors' common room] with all these people...I think with COVID you can't hang out with anyone anymore. We [got] told off for having lunch around two tables yesterday and even the opportunity to sit and relax with folk has disappeared. (T3I)

The ability to get to know others was also impaired due to teaching sessions being cancelled or changed to virtual formats which 'is very impersonal' (T61W) and they found that 'the [reduced] ability to network and meet people has impacted my training'. In addition, the wearing of masks provided another physical barrier to building relationships:

Not recognising people and working with people and there is anonymity, so you have got to make much more effort to introduce yourself to people...you are like 'I don't know who that was and I wouldn't recognise them if I saw them again.' (T73I)

Some trainees reflected on integrating redeployed staff into teams and inter-team working of staff who do not usually work together:

We had a lot of redeployed; I had a sexual health consultant, who shadowed me on a ward round which was a bit awkward and...there was a bit of a camaraderie, team effort and recognising each other's skills. (T52I)

\section{Culture and leadership}

There were some positive changes to the culture and trainees described a sense of camaraderie within the workplace through facing the pandemic alongside other healthcare professionals:

There is a bit more, everybody giving everyone an easier time. Everyone knows everyone is having a tough time, people are more forgiving as everybody is struggling a bit at the moment, the hospitals are in a bit of disarray, so they give each other an easier time. (T73I)

In keeping with the honeymoon phase of disaster, there was a sense of community cohesion within the workplace:
I think people are closer across these interprofessional groups, there's a recognition that maybe the less important things that would normally cause you to butt heads, what do they really matter? (T65W)

Leadership was not explicitly mentioned by trainees in reflecting on the pandemic's impact on their core needs, although there was some reflection on the actions of those in leadership roles:

There was a kind of top-down decision that everyone coming through the assessment unit would have, would either be full escalation, or they would have a DNACPR [Do Not Attempt CardioPulmonary Resuscitation], it was quite binary. (T3W)

They described that 'they [hospital management] told us we've got ours [rest area] til March' (T3W) and expressed a lack of appreciation due to such decisions:

Why don't they care about us until there's a pandemic happening...it's like we don't matter anymore. (T6W)

\section{Competence}

Workload

The complexities of dealing with COVID-19 patients added to the workload for IM trainees:

...huge pressure to move patients away from the screening ward as soon as they become negative or positive, depending on where they should go...sometimes there were pressures from bed management for us to sort that out (T2W)

In addition to the volume of patients and the perception that 'upstairs was catching fire because of COVID19' (T42W), the increased workload associated with frequently-changing protocols was hard to keep up with:

I know you have got a protocol. The protocol is probably now out of date, because it's the next week, and things have got a lot worse (T44W)

Trainees also reflected a lack of variety in their workload and a perception of a lack of consideration of broader differential diagnoses:

But people became lazy...so they might have thought they were short of breath, but actually they were in heart failure, and the person just said, they're COVID. (T27W)

\section{Management and supervision}

Through recognition that the pandemic was a strain on everyone, some trainees described informal support from registrars:

The regs [registrars] took initially...two or three trainees in a group... just to update and see if there's any problems at work (T59W) 
In general, IM trainees did not describe a lack of supervision in spite of the pressures of the pandemic, but there were some individual trainees who found support lacking:

I've just been met with, 'Well done, we're sorry'. I appreciate sometimes it's unavoidable... we don't feel particularly well supported (T65W)

\section{Learning, training and development}

Due to the COVID-19 pandemic, many training sessions were either cancelled or changed to a virtual format. Due to the virtual nature of the sessions, some trainees found themselves accessing the online content from the hospital which hindered their opportunities for protected training time:

It's meant to be protected, but it's not protected because you're on the ward doing it and it's just a nightmare. (T62W)

However, some trainees found that they were supported to attend educational sessions remotely to obtain the 'bleep-free, interruption-free time' (T62W):

We will be allowed to go home in the afternoon, for teaching in the afternoon and do it from home. (T65W)

Trainees' opportunities to attend outpatient clinics as part of their training and curricular requirements were diminished:

Obviously with COVID especially, getting yourself sort of slotted into a face to face clinic...it's really hard to arrange. (T28W)

In addition, their Membership of the Royal College of Physicians exams were cancelled or rearranged and the challenges of studying were a cause for concern:

The COVID situation does concern me...feeling quite so tired sometimes, you know, how am I going to have the energy to revise for it? So it is a worry. (T11I)

\section{DISCUSSION}

This study used a combined observational and interview approach to explore the impact of the COVID-19 pandemic on IM trainees' core workplace needs. It highlights the way in which the COVID-19 pandemic has influenced every aspect of IM trainees' core workplace needs.

The GMC report, Caring for doctors, caring for patients outlined immediate steps and calls to action in 2019, some of which were fortuitously expedited in response to the COVID-19 pandemic. ${ }^{16}$ The action plan included an aim to give doctors control over their working lives, including providing minimum requirement work conditions. ${ }^{16}$ The need to provide doctors with rest facilities and access to food in healthcare organisations has previously been outlined in the BMA's Fatigue and Facilities charter in 2018, and the
COVID-19 pandemic brought some of these recommendations to fruition. ${ }^{20}$ Hospitals created dedicated rest areas, some supported by Project Wingman, volunteer airline crew from across the UK, with 'hot drinks on tap. ${ }^{21}$ IM trainees reflected on how 'having a non-clinical area to take breaks in' positively impacted their well-being during the pandemic, and also discussed the negative impact on their sense of value in the organisation when such facilities were subsequently removed. The action plan from the GMC report also aimed to help doctors feel valued, respected and supported, and have a sense of belonging. ${ }^{16}$ At the outset of the pandemic, the sense of identity of 'healthcare worker' was emphasised and the associated camaraderie fostered some improved intergroup relations, with trainees describing a sense of being a 'cohesive team'. We must now consider how we can maintain this rhetoric and sense of teamwork in the longer term, when not relying on the initial phases of disaster to facilitate cohesion. The GMC report highlights a need for compassionate leadership with shared values, the benefits of which have now been emphasised through the trainee voices in this study. ${ }^{16}$ Others have echoed the need to build on the learning from the pandemic, in particular drawing from positive examples of adapting in response to crisis and team building with peer support in challenging times. ${ }^{22} 23$

Trainees described other core needs that were neglected, with a loss of control over working lives and rotas. The uncertainty the COVID-19 pandemic brought, both clinically and relating to work conditions, was a source of significant anxiety for trainees. The anxiety of healthcare workers in the context of the pandemic has been echoed throughout the world. ${ }^{24}$ The loss of control and uncertainty has been experienced by society more widely with mass quarantine and associated mental health sequallae. ${ }^{25}$ It is therefore not surprising that uncertainty was an addition to the original framework described in this context, confirming the importance of a sense of control on our personal well-being. Other negative impacts were that of reduced training opportunities, particularly clinics and protected non-clinical teaching time. The COVID-19 pandemic experience of trainees has underlined the importance of the three areas of core needs highlighted in the GMC report. ${ }^{16}$ The trainees' reflections support the need, more so now than ever, for the initial calls to action to continue to be implemented as we navigate our working lives in an adjusted workplace in the wake of the pandemic.

\section{Study strengths, limitations and future work}

This study accessed a national sample of IM trainees, providing an illuminating overview of the experiences of IM trainees in Scotland. This study explores the perspectives of IM trainees, but we know the COVID-19 pandemic has caused disruption across various specialties. ${ }^{222-29}$ The ABC framework applies to all trainee doctors and should be of interest and value to all trainees and trainers. ${ }^{16}$ Future studies could focus on interventions to support the autonomy, belonging and competence of doctors, 
particularly reviewing the calls to action from the initial GMC report in 2019, and the progress made during the turbulent year following the report's publication. ${ }^{16}$

\section{CONCLUSIONS}

Using the $\mathrm{ABC}$ of doctor's core needs as a conceptual framework for this study highlighted the impact of the COVID-19 pandemic on all domains for IM trainees in Scotland. ${ }^{16}$ It has highlighted an opportunity to foster the renewed sense of camaraderie among healthcare teams, while rebuilding work conditions to support autonomy and competence by supporting workplace learning for trainees.

\section{Twitter Joanne Kerins @JoanneKerins}

Acknowledgements The authors thank all of the trainee participants, all of the faculty involved in the IM boot camp, Julie Mardon and Tanya Somerville for their support and provision of access to the Scottish Centre for Simulation and Clinical Human Factors for this research, and First Class Secretarial Transcripion Services and Elizabeth Johnstone for their assistance in the transcription of the data.

Contributors JK the conception and the design of the study, data collection, analysis and interpretation of the data and the drafting and the revision of the manuscript. ALH and VRT contributed to the conception and design of the study, the analysis and interpretation of the data and the drafting and the revision of the paper. JP and FF contributed to the conception and the design of the study, data collection and revision of the paper. All authors (JK, ALH, JP, FF and VRT) approved the final manuscript for publication and have agreed to be accountable for all aspects of the work.

Funding This work was supported by a small grant award from the Scottish Medical Education Research Consortium (SMERC) for which we are extremely grateful.

\section{Competing interests None declared.}

Patient and public involvement Patients and/or the public were not involved in the design, or conduct, or reporting, or dissemination plans of this research.

\section{Patient consent for publication Not required.}

Ethics approval This study received ethical approval from the NHS Education for Scotland ethics review board with reference number NES/Res/14/20/Med. All participants gave written consent for data collection and the publication of anonymised results. Participants were free to leave the study at any time without giving a reason.

Provenance and peer review Not commissioned; externally peer reviewed.

Data availability statement Data are available on reasonable request. Raw transcripts of the data analysed in the study are available from the lead author.

Open access This is an open access article distributed in accordance with the Creative Commons Attribution Non Commercial (CC BY-NC 4.0) license, which permits others to distribute, remix, adapt, build upon this work non-commercially, and license their derivative works on different terms, provided the original work is properly cited, appropriate credit is given, any changes made indicated, and the use is non-commercial. See: http://creativecommons.org/licenses/by-nc/4.0/.

\section{ORCID iD}

Joanne Kerins http://orcid.org/0000-0001-9558-849X

\section{REFERENCES}

1 General Medical Council. National training survey 2020: summary of results. Londdon: GMC Publ, 2020: 1-5. https://www.gmc-uk.org/-/ media/documents/nts-results-2020-summary-report_pdf-84390984. pdf

2 Pillai S, Siddika N, Hoque Apu E, et al. COVID-19: situation of European countries so far. Arch Med Res 2020;51:723-5.

3 Rimmer A. Covid-19: most trainees have faced disruption to their training, GMC survey shows. BMJ 2020;371:m4093.
4 Fonseka TR, Ellis RJ. The personal impact of covid-19 on trainees. BMJ 2020;371:m4093.

5 Yuen J, Xie F. Medical education during the COVID-19 pandemic: perspectives from UK trainees. Postgrad Med J 2020;96:432-3.

6 Shih KC, Chan JC-H, Chen JY, et al. Ophthalmic clinical skills teaching in the time of COVID-19: a crisis and opportunity. Med Educ 2020;54:663-4.

7 Blecher GE, Blashki GA, Judkins S. Crisis as opportunity: how COVID-19 can reshape the Australian health system. Med J Aust 2020;213:196-8.

8 Helmich RC, Bloem BR. The impact of the COVID-19 pandemic on Parkinson's disease: hidden Sorrows and emerging opportunities. $J$ Parkinsons Dis 2020;10:351-4.

9 Gaur U, Majumder MAA, Sa B, et al. Challenges and opportunities of preclinical medical education: COVID-19 crisis and beyond. SN Compr Clin Med 2020;2:1992-7.

10 Ball CG. COVID-19: a time of crisis, but also of surgical opportunity and optimism. Can J Surg 2020;63:E164.

11 Wright HM, Griffin BJ, Shoji K, et al. Pandemic-related mental health risk among front line personnel. J Psychiatr Res 2021;137:673-80.

12 DeWolfe DJ. Training manual for mental health and human service workers in major disasters. Second Edition. Washington, DC: US Department of Health and Human Services, Substance Abuse and Mental Health Services Administration, Center for Mental Health Services, 2000. https://eric.ed.gov/?id=ED417056

13 SAMSHA (Substance Abuse and Mental Health Services Health Administration). Phases of disaster. U.S. department of health and human services, 2020. Available: https://www.samhsa.gov/dtac/ recovering-disasters/phases-disaster

14 Myers D. Key concepts of disaster mental health. In: disaster response and recovery: a Handbook for mental health professionals. Collingdale, PA: DIANE Publishing, 1994: 1-155.

15 Young BH, Ford JD, Friedman MJ. Disaster mental health services a guidebook for clinicians and administrators, 2008: 1-2.

16 West M, Coia D. Caring for doctors caring for patients. London: General Medical Council, 2019. https://www.gmc-uk.org/about/ how-we-work/corporate-strategy-plans-and-impact/supportinga-profession-under-pressure/uk-wide-review-of-doctors-andmedical-students-wellbeing\%0Ahttps://www.gmc-uk.org/-/media/ documents/caring-for-doctors-caring-for-patients

17 Paterson BL, Bottorff JL, Hewat R. Blending observational methods: possibilities, strategies, and challenges. Int J Qual Methods 2003;2:29-38.

18 King N. Template analysis. In: Symon G, Cassell C, eds. Qualitative methods and analysis in organizational research: a practical guide. London: SAGE Publications, 1998: 118-34.

19 NHS Employers, British Medical Association. Good Rostering guide, 2018. Available: https://www.bma.org.uk/-/media/files/pdfs/ employment advice/contracts/junior doctor contract/bma-nhsegood-rostering-guidance-may2018.pdf?la=en

20 BMA. Bma fatigue and facilities charter. Br Med Assoc, 2018. Available: https://www.bma.org.uk/media/1076/bma-fatigue-andfacilities-charter july2018.pdf

21 Glasper A. Strategies to protect the emotional health of frontline NHS staff in the pandemic. Br J Nurs 2021;30:252-3.

22 Anwar A, Seger C, Tollefson A, et al. Medical education in the COVID-19 era: impact on anesthesiology trainees. J Clin Anesth 2020;66:109949.

23 Behrman S, Baruch N, Stegen G. Peer support for junior doctors: a positive outcome of the COVID-19 pandemic? Future Healthc $J$ 2020;7:e64-6.

24 Vindrola-Padros C, Andrews L, Dowrick A, et al. Perceptions and experiences of healthcare workers during the COVID-19 pandemic in the UK. BMJ Open 2020;10:e040503-8.

25 Usher K, Durkin J, Bhullar N. The COVID-19 pandemic and mental health impacts. Int J Ment Health Nurs 2020;29:315-8.

26 Rimmer MP, AI Wattar BH, UKARCOG Members. Provision of obstetrics and gynaecology services during the COVID-19 pandemic: a survey of junior doctors in the UK National health service. BJOG 2020;127:1123-8.

27 Coughlan C, Nafde C, Khodatars S, et al. COVID-19: lessons for junior doctors redeployed to critical care. Postgrad Med $\mathrm{J}$ 2021;97:188-91.

28 James HK, Pattison GTR. Disruption to surgical training during Covid-19 in the United States, United Kingdom, Canada, and Australasia: a rapid review of impact and mitigation efforts. J Surg Educ 2021;78:308-14.

29 Hourston GJM. The impact of despecialisation and redeployment on surgical training in the midst of the COVID-19 pandemic. Int $J$ Surg 2020;78:1-2. 